\title{
Role of the anterior insula in task-level control and focal attention
}

\author{
Steven M. Nelson • Nico U. F. Dosenbach • \\ Alexander L. Cohen - Mark E. Wheeler • \\ Bradley L. Schlaggar · Steven E. Petersen
}

Received: 8 January 2010/Accepted: 21 April 2010/Published online: 29 May 2010

(C) The Author(s) 2010. This article is published with open access at Springerlink.com

\begin{abstract}
In humans, the anterior insula (aI) has been the topic of considerable research and ascribed a vast number of functional properties by way of neuroimaging and lesion studies. Here, we argue that the aI, at least in part, plays a role in domain-general attentional control and highlight studies (Dosenbach et al. 2006; Dosenbach et al. 2007)
\end{abstract}

Electronic supplementary material The online version of this article (doi:10.1007/s00429-010-0260-2) contains supplementary material, which is available to authorized users.

S. M. Nelson $(\bowtie) \cdot$ N. U. F. Dosenbach ·

A. L. Cohen - B. L. Schlaggar - S. E. Petersen

Department of Neurology, School of Medicine,

Washington University, Room 2220, 4525 Scott Ave,

Saint Louis, MO 63110, USA

e-mail: stevenelson@wustl.edu

\section{E. Wheeler}

Department of Psychology, Learning Research and Development

Center, University of Pittsburgh, Pittsburgh, PA, USA

M. E. Wheeler

Center for the Neural Basis of Cognition,

University of Pittsburgh, Pittsburgh, PA, USA

B. L. Schlaggar · S. E. Petersen

Department of Radiology, Washington University,

Saint Louis, MO, USA

B. L. Schlaggar · S. E. Petersen

Department of Anatomy and Neurobiology,

Washington University, Saint Louis, MO, USA

B. L. Schlaggar

Department of Pediatrics, Washington University,

Saint Louis, MO, USA

S. E. Petersen

Department of Psychology, Washington University,

Saint Louis, MO, USA supporting this view. Additionally, we discuss a study (Ploran et al. 2007) that implicates aI in processes related to the capture of focal attention. Task-level control and focal attention may or may not reflect information processing supported by a single functional area (within the aI). Therefore, we apply a novel technique (Cohen et al. 2008) that utilizes resting state functional connectivity MRI (rs-fcMRI) to determine whether separable regions exist within the aI. rs-fcMRI mapping suggests that the ventral portion of the aI is distinguishable from more dorsal/anterior regions, which are themselves distinct from more posterior parts of the aI. When these regions are applied to functional MRI (fMRI) data, the ventral and dorsal/anterior regions support processes potentially related to both task-level control and focal attention, whereas the more posterior aI regions did not. These findings suggest that there exists some functional heterogeneity within aI that may subserve related but distinct types of higher-order cognitive processing.

Keywords Insula - Control - Focal attention . Functional connectivity $\cdot$ fMRI $\cdot$ fcMRI

\section{Introduction}

The insula comprises a large swath of cortex hidden beneath temporal, frontal, and parietal cortex. It comprises the fundus of the lateral/Sylvian fissure, extending from the frontal operculum to the supramarginal gyrus. This article focuses on the anterior-most portion of the insula, which is anatomically and functionally distinct from the remainder of the insula.

In macaque, the anterior insula (aI) has been dissociated from the middle and posterior insula based on its 
cytoarchitecture and connectional anatomy. The macaque aI receives gustatory, olfactory, autonomic, and somesthetic inputs. Cytoarchitectonically, the macaque aI is continuous with adjacent dysgranular orbitofrontal cortex, but clearly distinct from more posterior granular insular cortex (Mesulam and Mufson 1982a, b; Mufson and Mesulam 1982).

While the human aI is also clearly separable from the remainder of the insula, it appears to have no macaque equivalent (Craig 2009). In the human insula, primary interoceptive representations such as autonomic and gustatory processing extend from the posterior to the middle insula, while they encompass the entire insula, including the aI, in macaques (Craig 2009). Furthermore, portions of the human aI contain von Economo neurons, also known as spindle neurons, which are layer $\mathrm{V}$ projection neurons that have a bipolar instead of a pyramidal shape. These neurons are more densely represented in hominids (and some cetaceans and elephants) and appear to be found most densely in the anterior insula and the anterior cingulate cortex (Allman et al. 2005; Butti and Hof 2010). In primates, these von Economo neurons are scarce in macaques, more abundant in apes and found in greatest numbers in humans (Nimchinsky et al. 1999; Allman et al. 2005, 2010). The remainder of this article will deal specifically with the structure and function of the human aI.

Neuroimaging studies targeting the aI in humans, in part inspired by findings in macaques, have been focused on its possible role in sensing and controlling autonomic processes such as heart rate (Gray et al. 2009), bladder (Kavia et al. 2005), and bowel distension (Dunckley et al. 2005), pain (Albanese et al. 2007), and empathy for pain (Lamm et al. 2010), among others. As purported, the aI was found to be active in viscerosensory and autonomic control tasks. However, with an explosion in the number of functional neuroimaging studies, it soon became evident that the aI was also active across a wide variety of tasks with no obvious ties to autonomic and interoceptive processes. These behavioral manipulations have included, but are not limited to, studies of the neurophysiology of reward (Linke et al. 2010), dread (Berns et al. 2006), disappointment and regret (Chua et al. 2009), social rejection (Eisenberger et al. 2003), errors (Hester et al. 2004), error awareness (Klein et al. 2007), and decision-making (Thielscher and Pessoa 2007).

Using a meta-analytic approach, Duncan et al. (2000) were among the first to point out that several brain regions, including the anterior insula/frontal operculum (aI/fO), seem to be active across disparate processing domains such as language and mathematical reasoning (Duncan and Owen 2000). A subsequent meta-analysis of task-level control signals (see below) across 10 tasks, also with no obvious connections to visceral control or sensation, showed the aI and dorsal anterior cingulate cortex (dACC) to be most commonly activated across tasks (Dosenbach et al. 2006). Since then, the collection of manipulations that have found differential aI activation has continued to grow rapidly.

Importantly, a database of published foci, i.e., stereotactic coordinates of functional involvement, and tools for visualizing thousands of foci from a myriad of imaging studies have been developed by Van Essen and colleagues (Van Essen 2009; Van Essen et al. 2001; Van Essen and Dierker 2007). When using these tools to plot 41,581 foci from 1,114 studies published in the journals Neuron (20022008), Nature Neuroscience (2001-2008), Proceedings of the National Academy of Sciences (2006-2008), Journal of Neuroscience (1996-2008), and Cerebral Cortex (19972007) as a foci density map, it became evident that the left and right aI are among the most commonly activated brain regions across all tasks (Fig. 1). As previously highlighted (Duncan and Owen 2000; Dosenbach et al. 2006; Craig 2009), this analysis showed a similar high density of reported foci in the dACC.

On one hand, it seems that the aI has been ascribed specific roles in many very distinct tasks that span a large range of cognitive processes. On the other hand, the metaanalytic data suggest that the aI may have a significantly more general role in attention and task-level control. Thus, one could either argue that the aI contains a large number of small separate functional entities that carry out separate processes, or one might interpret these findings as an indication that the aI carries out basic computational processes shared across most goal-oriented behaviors. As we will discuss in the succeeding sections, our data favor the second interpretation, while providing some evidence for functional heterogeneity as well.

As support, we will highlight previous work (Dosenbach et al. 2006; Dosenbach et al. 2007; Ploran et al. 2007; Dosenbach et al. 2008) that provides evidence for aI regions contributing to general attention and control processes. In subsequent sections, we will present results from a resting-state functional connectivity (rs-fcMRI) mapping study that confirms the presence of several separate functional regions within the insula, and suggests further distinctions within the aI as well. We will then present further evidence that while some of the functional separations found in the aI likely subserve general attention and taskcontrol processes, others do not appear to be related to these more general processes.

\section{Part of the aI plays a general role in task-level control, performance monitoring, and focal attention capture}

In previous work, we have argued that the aI is important for the implementation of task sets across a wide range of 
Fig. 1 Activation foci in bilateral anterior insula (aI) are among the most commonly found in functional neuroimaging studies. Regions of interest from 41,581 foci across 1,114 studies were used to create a foci density plot, shown here on inflated PALS atlas cortical surfaces (Caret). The map represents how often a region of interest has been reported at a particular location. Studies containing these foci were reported in the journals Neuron (2002-2008), Nature Neuroscience (2001-2008), Proceedings of the National Academy of Sciences (20062008), Journal of Neuroscience (1996-2008), and Cerebral Cortex (1997-2007). The locations of the foci from these studies can be accessed at http://brainvis.wustl.edu

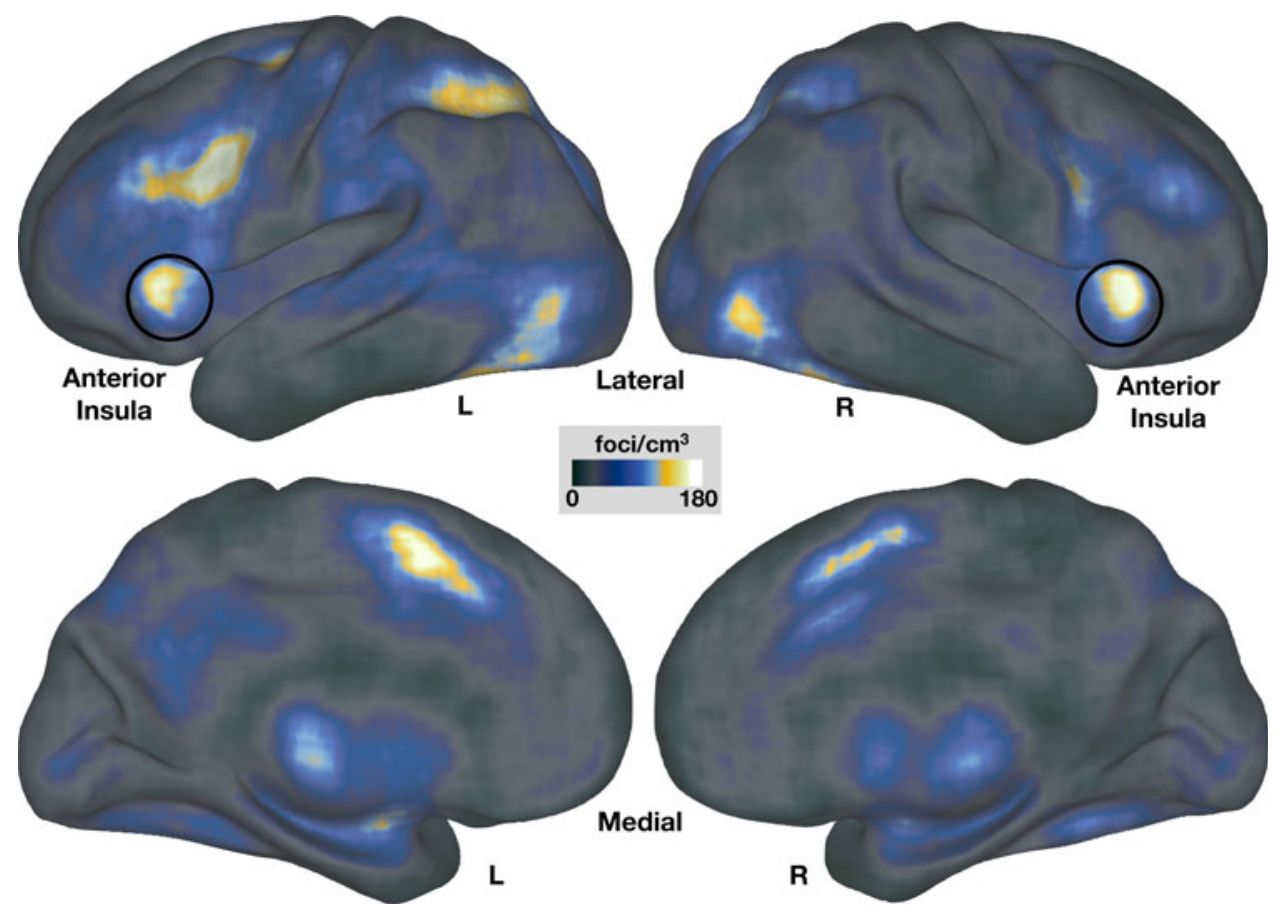

goal-directed tasks (Dosenbach et al. 2008). Under specific task demands, moment-to-moment processes combining sensory inputs, cognitive categorizations, and motor outputs must be configured. To do so, humans are thought to enter a task set that is maintained for the duration of the task. Mixed blocked/event-related designs allow task setrelated signals to be extracted from fMRI experiments (Visscher et al. 2003), including activity related to cues that signal the beginning of a task block (related to initializing task goals and parameters), "set-maintenance" activity sustained for the duration of a task block (related to maintaining goals and parameters), and event-related performance feedback signals (related to adjusting ongoing task performance and/or learning to perform similar tasks more efficiently in the future) (Fig. 2).

Previously, we analyzed mixed blocked/event-related fMRI data from 10 different tasks (Dosenbach et al. 2006), and found that the bilateral aI showed reliable start-cue and sustained activations in nearly all tasks. The aI also contained the most reliable error-related feedback signals in a subset of tasks (Ullsperger et al. 2010). The only other brain region with this activation pattern (across the majority of the tasks) was the dACC. These findings lead us to suggest that the aI and AACC together form a "core" system for the implementation of task sets (Dosenbach et al. 2006).

We followed the meta-analyses of task control signals by studying rs-fcMRI correlations between all the regions that showed any of the aforementioned signal types (onset of task, maintenance and errors). These rs-fcMRI analyses also showed the aI to be strongly functionally connected to the dACC (Dosenbach et al. 2007). The aI and dACC formed the core of a larger "cingulo-opercular" control network that also included specific regions in anterior prefrontal cortex (aPFC) (Fig. 3, black). Additionally, this analysis suggested the dissociation of these regions from a "fronto-parietal" control network that included the dorsolateral prefrontal cortex (dlPFC) and intraparietal sulcus (IPS) (Fig. 3, yellow). The fronto-parietal network emphasized start-cue and error-related activity, hence we proposed that the frontoparietal network may initiate task set and adapt control on a trial-by-trial basis, whereas the cingulo-opercular network may have special importance for the maintenance of task sets across task epochs (Sterzer and Kleinschmidt 2010).

In a separate study (Ploran et al. 2007), we investigated perceptual recognition processes using an extended reveal paradigm. This paradigm allowed us to examine the time course of brain activity as an object was revealed from a mask of noise over the course of $16 \mathrm{~s}$ (Fig. 4a). The slow nature with which the reveal took place afforded us increased temporal resolution in matching the BOLD response to ongoing processing and behavior, and made it possible to dissociate activity related to different stages of recognition. Prior to the time of recognition, information about the stimulus is being gathered and processed, but is insufficient to elicit commitment to identification. At some point, the accumulated information reaches a critical threshold wherein the subject feels confident that they can identify the object.

Among the signals we were able to dissociate were those in posterior visual regions that varied with the amount of visual information available. A set of frontal and parietal regions had a slow increase of activity that peaked with the 


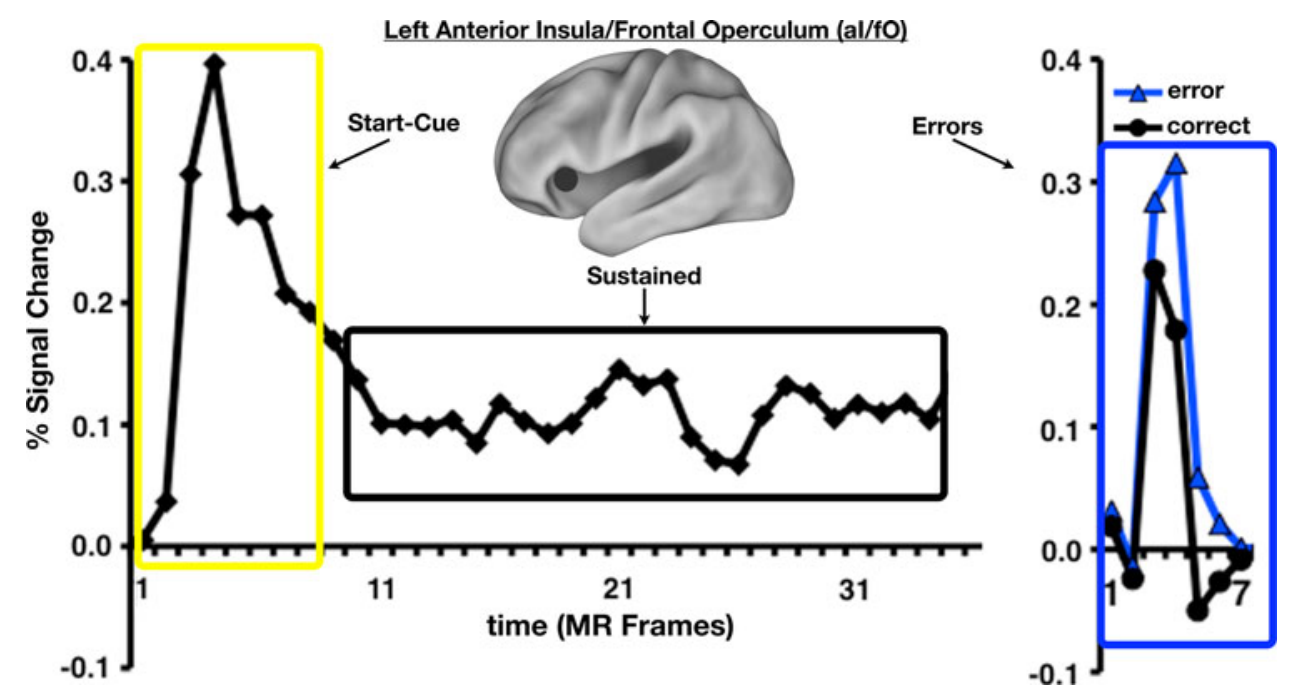

Fig. 2 Across a variety of tasks, bilateral anterior insula (aI) contains signals that are consistent with a general role in task-level control. The mixed blocked/event-related fMRI design allows the separate extraction of three different types of task set-related signals. Shown here is the average remaining task-related activity (left panel) after trial-related activity (right panel) has been extracted. Activity timelocked to the start of a task block (yellow) is likely important for the
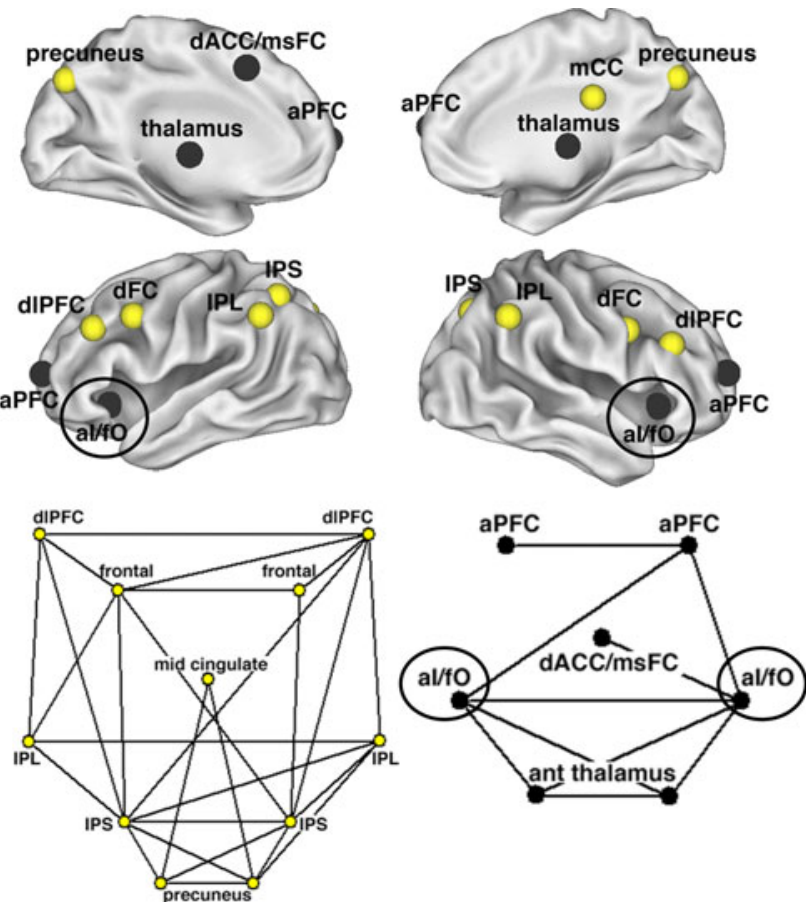

Fig. 3 The anterior insula (aI) forms part of a cingulo-opercular control network, distinct from a fronto-parietal control network. Human cingulo-opercular (black) and fronto-parietal (yellow) control networks displayed on inflated brain surfaces. The network structure of human control networks is displayed as a two-dimensional pseudoanatomical graph layout. Black lines indicate strong resting state functional connections between brain regions. Adapted from Proceedings of the National Academy of Sciences (Dosenbach et al. 2007). Copyright (2007), with permission from National Academy of Sciences, USA initiation of task sets, while activity sustained for the length of the task period (black) is likely related to the maintenance of task sets to ensure continued performance success. Error-related signals (blue) provide performance feedback on a trial-by-trial basis. Adapted from Neuron (Dosenbach et al. 2006). Copyright (2006), with permission from Elsevier

time of recognition, i.e., as if these regions were accumulating information toward recognition of the object. Whether the subject is developing hypotheses about the stimulus identity explicitly or implicitly, as less salient features of the stimulus are revealed, the number of possible responses begins to decrease over time. During this pre-response phase, regions in the bilateral aI and dACC show minimal or no activity but then peak transiently when the subjects feel they have identified the item.

Importantly, bilateral aI and a number of regions along the dACC showed a transient response with a late onset and a peak that shifted with the time of recognition. We postulate that these transient responses occurring at the moment of recognition are evidence that regions in the aI and AACC are involved in the capture of focal attention (i.e., when accumulated, processed information becomes available to awareness). It may also be possible that these regions subserve "feedback" processing related to the successful entry of a specific object into awareness (Wheeler et al. 2008).

The distinction between the capture of focal attention and feedback is perhaps a narrow one. In the former case, the response at the time of recognition could reflect the moment when the processed evidence about an object reaches a level that commits to the "a-ha" feeling of recognition. The observation of greater activity for errors than correct trials could also be related to focal attention, in that errors would entail a greater level of awareness of, or "thinking about", the trial than does a correctly performed trial. In the latter case, observed signals in the recognition 
Fig. 4 In an extended reveal paradigm, BOLD time course activity in bilateral anterior insula (aI) responds transiently at the moment of recognition. Objects were revealed from a mask of noise in $2 \mathrm{~s}$ steps and subjects made a button press when they could identify the object. At the end trial VoA (verification of accuracy) stage, the picture was fully revealed and subjects were asked to indicate by button press whether they had correctly identified the Average time courses are depicted for a set of regions that showed baseline activity until subjects responded that they recognized an object being unmasked from a noise field. The activity was time-locked to the subjects' response, resulting in a gradual shift in the onset depending upon what "step" or "reveal stage $\left(\mathrm{T}_{\mathrm{R}} 4,5,6,7\right)$ " the subject recognized the item. Adapted from Journal of Neuroscience (Ploran et al. 2007). Copyright (2007) with Neuroscience item as it was being revealed. permission from the Society for

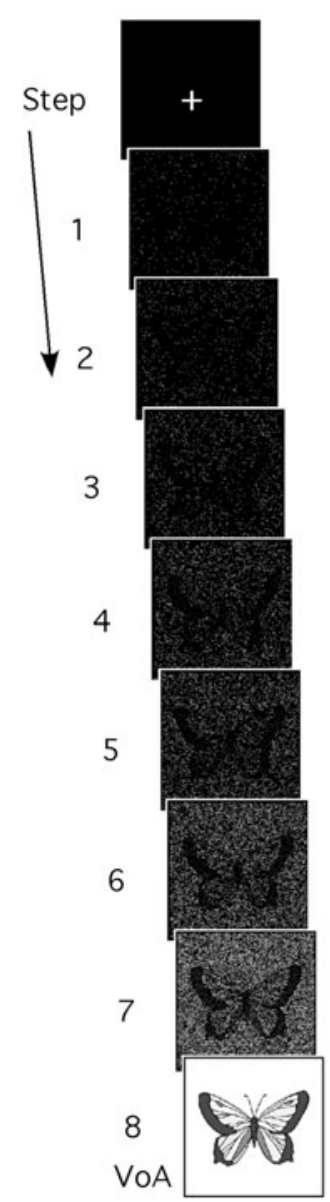

experiment could reflect post-"moment of awareness" evaluative feedback processes, similar to those distinguishing errors from correct trials (Wheeler et al. 2008). If in the same experiment, we could demonstrate regions that show clear error-like signals without the recognition-like profile of the Ploran et al. (2007) experiment, these regions could be more unequivocally ascribed to feedback processing rather than recognition per se. On the other hand, if regions failed to show error signals but depicted a recognition-like profile, it would leverage an argument for the focal attention hypothesis. We will present data from a new recognition experiment that could address the first of these possibilities in the final section.

\section{Methods and results: rs-fcMRI mapping}

rs-fcMRI mapping suggests a dissociation of the aI from the rest of the insula, and five putative functional areas within the aI

Recently, parcellation schemes for the aI have employed such techniques as diffusion weighted imaging (DWI) (Nanetti et al. 2009) and activation likelihood estimate
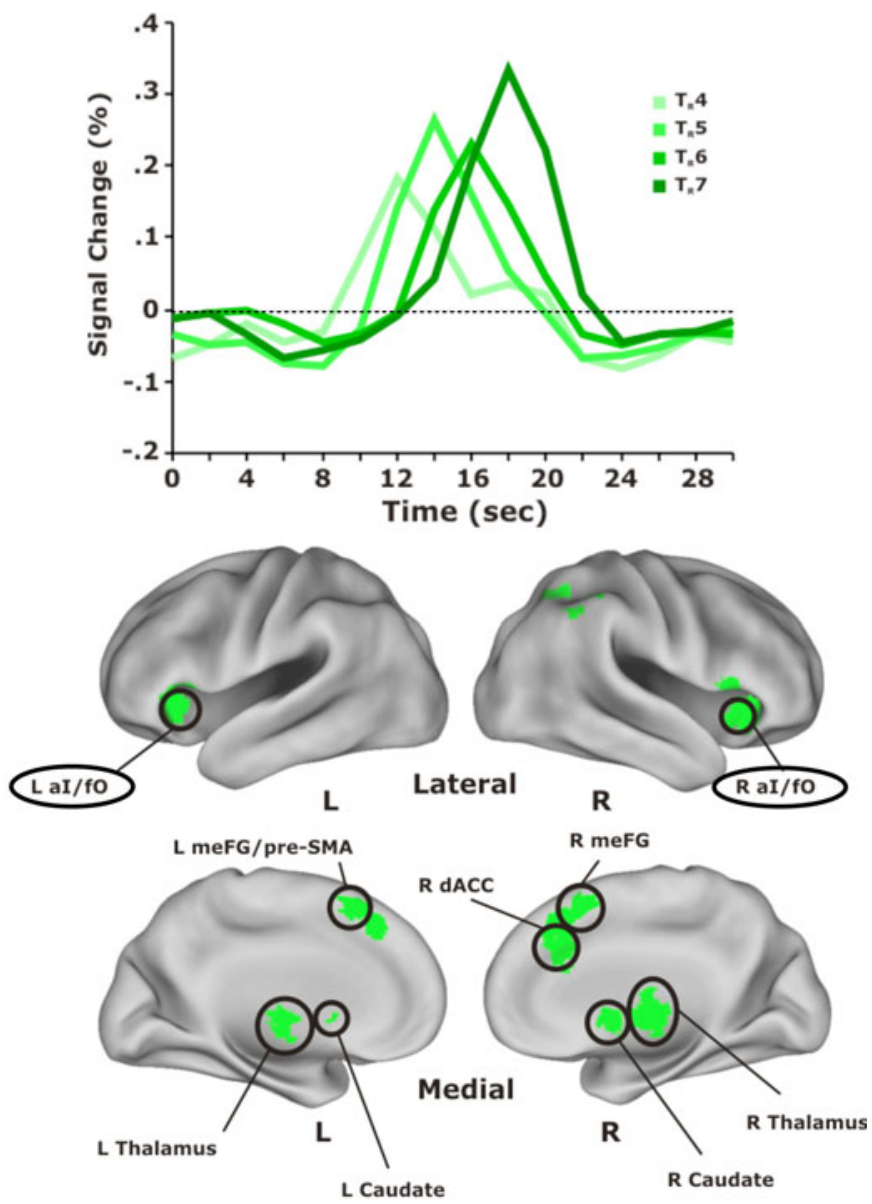

(ALE) (Mutschler et al. 2009). Cohen et al. (2008) described a technique that uses rs-fcMRI to define putative functional areas and this may also allow for a more finegrained functional parcellation within the aI. The discovery of multiple, distinct functional areas would provide a basis for determining whether processes related to control, performance monitoring, and focal attention, as well as other putative functional distinctions within the aI, are subserved by one or more functional areas. It should be noted that though the results described here relate to left aI, we performed the same set of analyses (both rs-fcMRI and fMRI) on the right aI and found highly similar results (see Supplemental Data).

\section{Methods}

\section{Subjects}

Both task-related fMRI and rs-fcMRI data were collected on a set of 21 subjects (11 female, age 21-28 years). rs-fcMRI analyses were conducted on all 21 subjects, and fMRI data analysis was conducted on a subset of 19 subjects (10 male, age 21-28 years) as 2 subjects provided an inadequate amount of data to appropriately quantify 
effects. This section describes the methods and results related to rs-fcMRI mapping of these data.

\section{$r s-f c M R I$ paradigm}

rs-fcMRI data were collected while subjects were instructed to relax and fixate on a white crosshair against a black background. Three runs (2.5 s TR, 133 frames per run) were acquired for each subject.

\section{Image acquisition}

Images were acquired in adherence to a standard protocol. To help stabilize head position, each subject was fitted with a thermoplastic mask fastened to holders on the head coil. All images were obtained with a Siemens MAGNETOM Tim Trio 3.0T Scanner (Erlangen, Germany) and a Siemens 12 channel Head Matrix Coil. A T1-weighted sagittal MP-RAGE structural image was obtained $(\mathrm{TE}=3.08 \mathrm{~ms}, \mathrm{TR}($ partition $)=2.4 \mathrm{~s}, \mathrm{TI}=1000 \mathrm{~ms}$, flip angle $=8^{\circ}, 176$ slices with $1 \times 1 \times 1 \mathrm{~mm}$ voxels $)(\mathrm{Mu}-$ gler and Brookeman 1990). A T2-weighted turbo spin echo structural image $(\mathrm{TE}=84 \mathrm{~ms}, \mathrm{TR}=6.8 \mathrm{~s}, 32$ slices with $2 \times 1 \times 4 \mathrm{~mm}$ voxels) in the same anatomical planes as the BOLD images was also obtained to improve alignment to an atlas. An auto align pulse sequence protocol provided in the Siemens software was used to align the acquisition slices of the functional scans parallel to the anterior commissure-posterior commissure (AC-PC) plane and centered on the brain. This plane is parallel to the slices in the Talairach atlas (Talairach and Tournoux 1988). Functional imaging was performed using a blood oxygenation leveldependent contrast sensitive gradient echo echo-planar sequence $\left(\mathrm{TE}=27 \mathrm{~ms}\right.$, flip angle $=90^{\circ}$, in-plane resolution $=4 \times 4 \mathrm{~mm})$. Whole brain EPI volumes $(\mathrm{MR}$ frames) of 32 contiguous, 4-mm thick axial slices were obtained every 2 or $2.5 \mathrm{~s}$.

\section{MR data preprocessing}

Imaging data from each subject were preprocessed to remove noise and artifacts, including (a) correction for movement within and across BOLD runs using a rigidbody rotation and translation algorithm (Snyder 1996), (b) whole brain intensity normalization to a common mode of 1,000 to allow comparisons across subjects (Ojemann et al. 1997), and (c) temporal realignment using sinc interpolation of all slices to the temporal midpoint of the first slice, accounting for differences in the acquisition time of each individual slice. Functional data were then resampled into $3 \mathrm{~mm}$ isotropic voxels and transformed into stereotaxic atlas space (Talairach and Tournoux 1988). Atlas registration involved aligning each subject's T1-weighted image to a custom atlas-transformed (Lancaster et al. 1995) target T1-weighted template using a series of affine transforms (Michelon et al. 2003; Fox et al. 2005a).

\section{Surface-based analysis}

The PALS atlas fiducial surface was transformed to the appropriate atlas space and used to obtain probable surface coordinates to apply to our group of subjects (Van Essen 2005). Using Caret software (Van Essen et al. 2001), a grid of seed points, or patch, was generated on the spherical PALS surface over the insula and the corresponding volumetric locations on an average fiducial surface in 711-2B atlas space were obtained.

The set of volumetric coordinates for a patch over the insula were used to generate a set of $3 \mathrm{~mm}$ diameter spherical regions of interest or seeds. For each seed, in each subject, volumetric correlation maps were generated as in Fox et al. (2005b) and Fair et al. (2007) by correlating the time course of this region of interest with the time courses of all other voxels over the entire volume of the brain within each subject. This approach creates a volumetric correlation map, for each seed in each subject, where the intensity at each voxel is the computed $z$-score of the Fisher Z-transformed correlation $(r)$ between that voxel's time course and the seed region's time course. To determine the similarity or difference between the correlation maps of two seed locations, eta ${ }^{2}$ matrices were calculated as in Cohen et al. (2008).

To find putative boundaries within this patch of cortex, the Canny edge detection algorithm (Canny 1986), as implemented in the Image Processing Toolbox (v7.2) of the MATLAB software suite, was applied to each seed's eta ${ }^{2}$ profile as in Cohen et al. (2008), and the resulting set of binary edge maps were averaged to generate an "rs-fcMRI boundary map" for each subject. The intensity at each location in the map represents the likelihood of a functional area boundary being located there, as indicated by a rapid transition in the underlying rs-fcMRI correlation maps. The average rs-fcMRI boundary map was created for the group of 21 subjects, producing a single map of the likelihood of functional area boundary and areal location across the group of 21 subjects.

Regions of interest that represent putative functional areas were created by applying straightforward 2D local extrema algorithms (MATLAB v7.2, Image Processing Toolbox, as well as custom-written software) to the rs-fcMRI boundary map. The corresponding volumetric coordinates for each identified putative area were then used to generate small spherical ROIs for further analyses as described below. 
Results

Performing rs-fcMRI mapping on a patch that encompassed the extent of the left insula, including the most anterior portion, defined 27 locations as putative functional areas (Fig. 5a). For our purposes, we will focus on the five most anterior ROIs defined within the patch residing in the left insula (Fig. 5b).

The distinctiveness of these putative areas is revealed by differences in their patterns of rs-fcMRI correlations. The boundaries defining these putative areas were based on the presence of rapid transitions in the underlying volumetric rs-fcMRI maps. Thus, the pattern of functional connections from each location should be distinct. One example of these differences lies in the relationship between the aI and the dACC. Shown in Fig. 6 are the five putative area locations in aI and their corresponding rs-fcMRI correlation maps
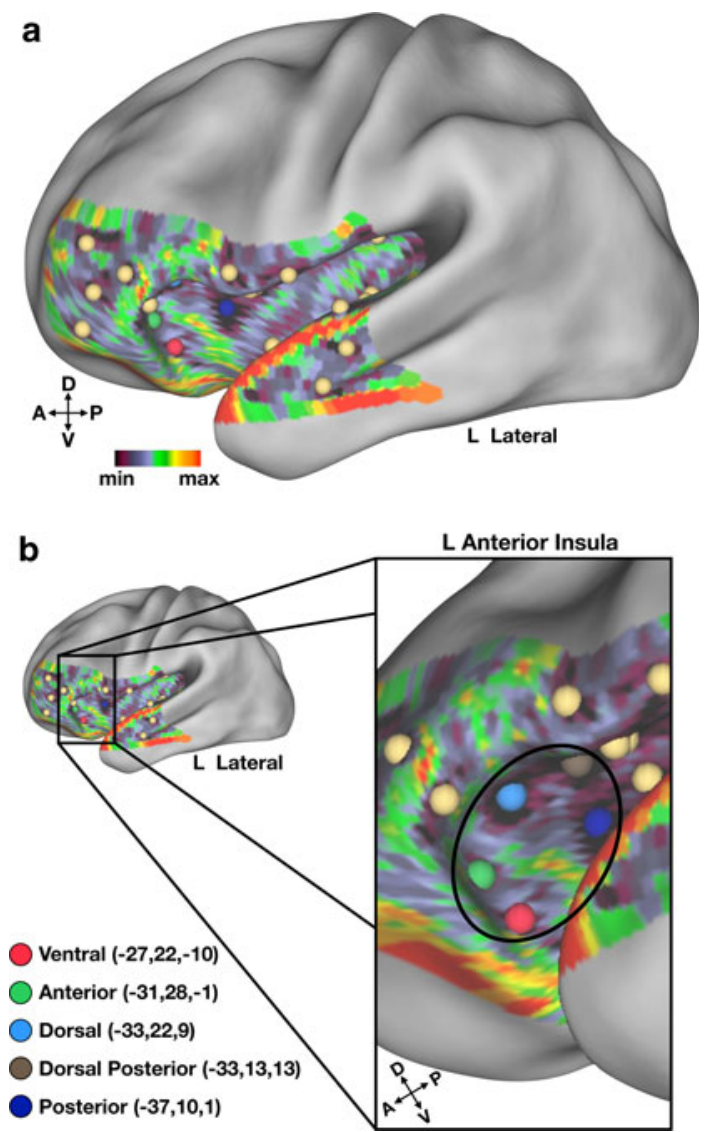

Fig. 5 rs-fcMRI mapping reveals locations in left anterior insula (aI) that may represent dissociable putative functional areas. a A grid of foci was placed over the extent of the left insula and surrounding cortex. 27 putative areas were defined in this "patch", including five in the anteriormost portion of the left insula. Spheres indicating regions outside of the al are colored in $\tan D$ (dorsal), $V$ (ventral), $A$ (anterior), $P$ (posterior). b A portion of the left hemisphere surrounding the aI is enlarged to depict the five locations defined therein (black circle). Regions are separately colored and named according to their relative locations within the aI. Stereotactic coordinates are reported in MNI space obtained from each seed location. The region in ventral aI (Fig. 6c) shows a very distinct pattern of correlation with $\mathrm{dACC}$ with strong correlations more ventral and anterior to those seen in the other aI correlation maps. A second region in the most anterior portion of the aI (Fig. 6b) reveals a pattern of correlation that also appears to be distinct, with strong correlations residing in the dorsal-most extent of dACC. The other three ROIs (Fig. 6a, d, e) appear to have highly overlapping patterns of correlation with dACC that are somewhat distinct from the anterior ROI (Fig. 6b) and clearly distinct from the ventral ROI (Fig. 6c).

\section{Methods and results: fMRI study}

fMRI study shows dissociations between aI regions exhibiting error and recognition-like responses and those that exhibit neither

We then applied the five aI ROIs to a study similar to that described in Ploran et al. (2007), in which sudden insight regarding a decision occurs as the subjects become aware of specific stimulus features.

\section{Methods}

\section{Behavioral paradigm}

The encoding task consisted of two unscanned runs in which the subjects were asked to make a living/non-living judgment on a randomly chosen set of 100 modified Snodgrass pictures (Rossion and Pourtois 2004). Stimuli appeared for $1.5 \mathrm{~s}$ and were presented every $2.5 \mathrm{~s}$. Subjects were not given explicit instructions that they would need to remember this material at any time in the future, but were simply told to decide if the presented object was living or non-living.

After encoding, subjects took part in a retrieval task (now scanned) where they were asked to determine if the picture was "old" or "new", depending on whether they had seen it during the encoding phase of the experiment (old) or had not (new).

At trial onset, pictures were covered by a black mask that partially dissolved at each successive $2 \mathrm{~s}$ interval (i.e., revelation step) until pictures were completely revealed. Participants were instructed to press a button when they could determine whether the item was old or new. Neither speed nor accuracy was emphasized in the response, and participants were not specifically encouraged to respond prior to full revelation.

Participants were asked to make an old/new judgment while the object was being unmasked. When stimuli were unmasked, subjects were instructed that they could make 


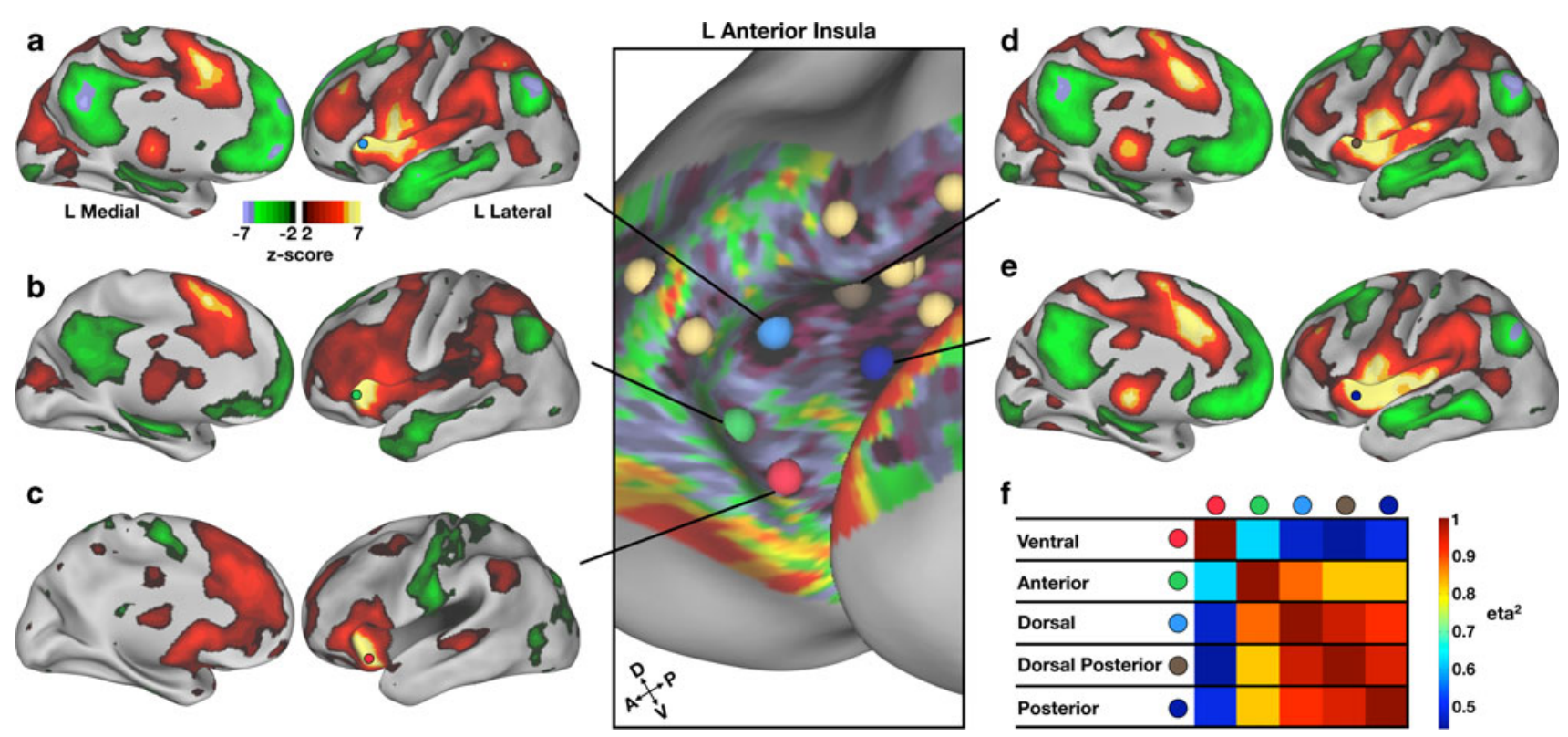

Fig. 6 An examination of the rs-fcMRI correlation patterns of the five putative area locations in left anterior insula (aI) reveals some distinct and overlapping patterns of functional connections. a-e rs-fcMRI correlation maps depicting regions of significant positive (dark red to yellow, $z$-score $>2.00$ ) and negative (dark green to blue, $z$-score $<-2.00$ ) correlations with each seed are shown on left lateral and medial inflated cortical surfaces (Caret). The

the same response as they had done during unmasking or could change their response.

\section{fMRI data analysis}

Pre-processed data were analyzed at the voxel level using a general linear model (GLM) approach (Friston et al. 1994; Miezin et al. 2000). Details of this procedure are described by Ollinger et al. (2001). Briefly, the model treats the data at each time point (in each voxel) as the sum of all effects present at that time point. Effects can be produced by events in the model and error. Estimates of the time course of effects were derived from the model for each response category by coding time points as a set of delta functions immediately following onset of the coded event (Ollinger et al. 2001). 15 time points were modeled in the GLM for each event. Over each run, a trend term accounted for linear changes in signal, and a constant term was used to model the baseline signal. Event-related effects are described in terms of percent signal change, defined as signal magnitude divided by a constant term. This approach makes no assumptions about the shape of the BOLD response, but does assume that all events included in a category are associated with the same BOLD response (Ollinger et al. 2001). Thus, we could extract time courses without placing constraints on their shape. Image processing and analyses were carried out using in-house software written in IDL (Research Systems, Inc.). location of each seed is shown on the left aI view (center of figure) and also on the lateral surface of the correlation map. $\mathbf{f}$ The similarity between the rs-fcMRI correlation maps is quantified using eta ${ }^{2}$ and displayed as a matrix that represents the relationship between each region in the aI. Eta ${ }^{2}$ values approaching 1 are indicative of highly similar maps, whereas eta ${ }^{2}$ values near 0.5 indicate that the two maps are statistically independent from one another

Results

We found signals consistent with a role in focal attention and/or feedback in the anterior ROIs (Fig. 7a, b, c); ROIs located posteriorly (Fig. 7d, e) did not show consistent recognition-like responses. The region located in ventral aI (Fig. 7c) showed the clearest moment of recognition response, as described in Ploran et al. (2007), as a result of late onset of the responses. The other regions located anteriorly (Fig. 7b) and dorsally (Fig. 7a) showed responses that peaked at the time of the decision, but did not exhibit the late response onset seen in the more ventral (Fig. 7c) region.

To probe the relationship with possible feedback responses, we also examined error versus correct responses in the same five regions to assess spatial overlap between recognition-like activity and error signals (Fig. 8).

Error signals were observed in the most anterior regions (Fig. 8a, b, c), similar to those found by Wheeler et al. (2008). In all of these regions, the signals took the form of greater activation for errors than correct responses, and a prolonged error response to the early "detection" responses. There was no region that showed an isolated prolonged error response, leading to an easy interpretation of performance monitoring. As with the functional connectivity results, the most ventral region appeared to dissociate from 

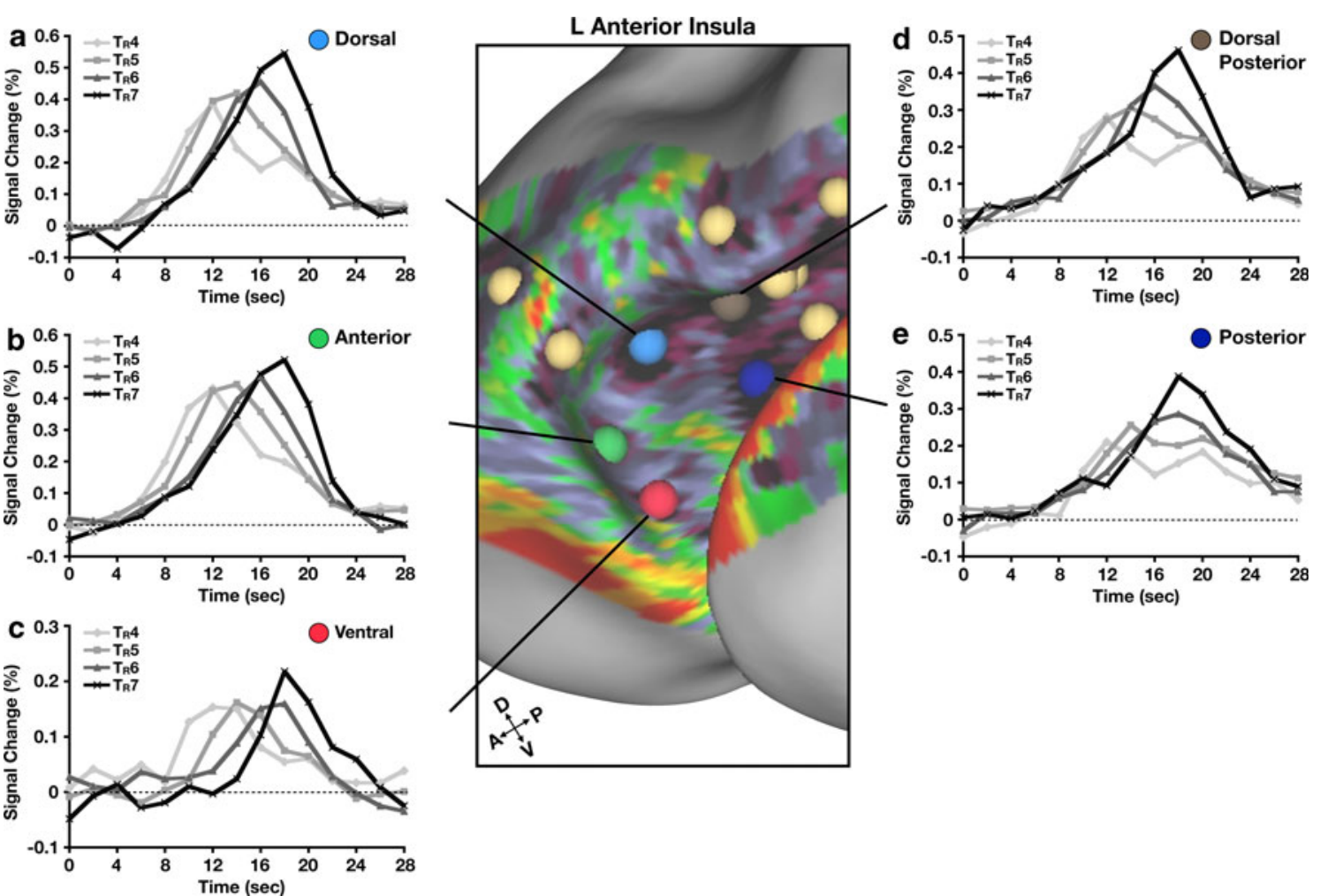

Fig. 7 Moment of recognition-like processing in anterior insula (aI). a-e For each region in aI, time courses were separately extracted for trials where the subject recognized the stimulus at each step of

the others, in this case on the basis of its late onset of activity.

Right aI showed a similar pattern of results across rs-fcMRI and fMRI-based analyses

While previous studies have pointed to functional asymmetries in the aI (Shaw et al. 2009; Houde et al. 2010), our data do not support lateralized differences (see Supplemental Data). Five regions were defined within right aI that mirrored those defined in left aI (Supp. Fig. 1) and the relationships between each region's rs-fcMRI correlation map showed a similar pattern of relationships as those defined in the left hemisphere (Supp. Fig. 2). Additionally, task-related fMRI data extracted from regions in the right aI resembled those in corresponding left aI regions (Supp. Fig. 3). The presence of error-related activity was observed in 4 of 5 right aI regions (Supp. Fig. 4), again mirroring the results seen in left aI.

\section{Conclusions}

The totality of the results presented here are consistent with the interpretation that the most anterior regions of aI take revelation, i.e., $T_{R} 4, T_{R} 5, T_{R} 6, T_{R} 7$, including only correct responses. Time courses are graphed in units of $\%$ signal change from baseline (dotted line) as a function of time in seconds

part in general processes involved in goal-oriented tasks. Additionally, there appear to be responses across an abundance of task conditions, related to either performance monitoring and/or focal attention. Without experiments expressly directed towards dissociating these two closely related information-processing accounts, neither processing ascription can yet be ruled out.

The relationship of some part of the most anterior regions of the aI to task-level control signals (i.e., the task initiation and task maintenance signals) also remains ambiguous as to its location. It is tempting to place it with one or more of the anterior regions found in this study, and the meta-analytic work is certainly consistent with this placement.

Our opinion, derived from the multiple converging lines of evidence discussed above, is that at least a significant portion of the aI is devoted to very general processes related to volitional top-down control, and to bottom-up signals related in some way to task-level control (Brass and Haggard 2010). It may be that conscious feedback drives adjustments in the top-down signals, and these adjustments could drive online processing or support future implementations of tasks with similar demands. On the other hand, some of the trial-related activity could actually represent information passing into awareness (Craig 2010) concerning the outcome of processing itself. Since there are 

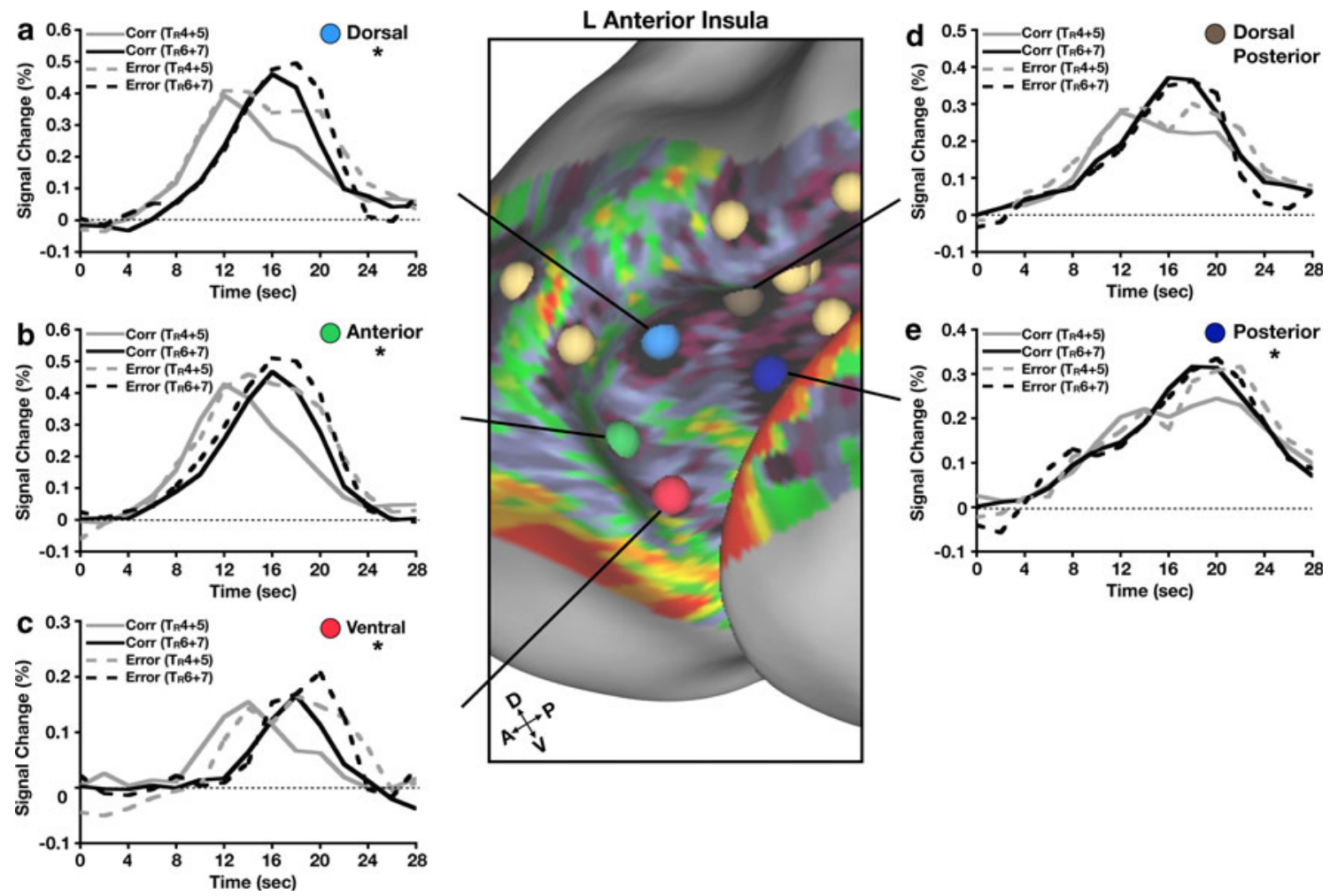

Fig. 8 Error processing in anterior insula (aI). a-e For each region in aI, time courses were extracted for correct responses (early $=$ $\mathrm{T}_{\mathrm{R}} 4+5$ and late $=\mathrm{T}_{\mathrm{R}} 6+7$ ) and errors (early $=\mathrm{T}_{\mathrm{R}} 4+5$ and late $=\mathrm{T}_{\mathrm{R}} 6+7$ ). Time courses are graphed in units of $\%$ signal

multiple regions showing these forms of activity, either explanation or both seem plausible.

As far as the multiplicity of explanations that have been made for aI activations over the past several years, some of these are plausibly subsumed under these more general processing ideas. This is particularly true for situations related to decision uncertainty, and other evaluative situations. Greater demand for evaluation of any kind would fit comfortably under either a performance monitoring or focal attention explanation.

For those results that do not comfortably fit, the presence of multiple regions within the aI does leave room for a limited number of dissociations. We seem to have three functionally distinct zones (Kurth et al. 2010) encompassing a dorsal/posterior aI putative area (Figs. 7a, d, e and 8a, d, e), an anterior aI putative area (Figs. $7 \mathrm{~b}, 8 \mathrm{~b}$ ), and a ventral aI putative area (Figs. 7c, 8c). These zones show clear differences in functional and/or functional connectivity profiles, and could thus subserve distinct sets of processes.

\section{What are the relative contributions of aI and dACC?}

So far it has not been possible to clearly dissociate the functional roles of the aI and dACC (Medford and

change from baseline (dotted line) as a function of time in seconds. A correctness $\times$ time repeated measures ANOVA was calculated for each region to identify effects of error vs correct responses. $* p<0.001$

Critchley 2010) in humans using neuroimaging. However, one might speculate on functional differences between the aI and dACC based on anatomical differences such as their respective descending projections to sensory and motor brainstem nuclei and the al's proximity to brain regions important for processing interoceptive information (Heimer and Van Hoesen 2006; Craig 2009). Perhaps one difference between the aI and the dACC is the kind of input and feedback they receive. The aI may emphasize, in relative terms, processing of internal visceral and motivational information relevant for consciously controlled behavior, such as autonomic inputs, fear, emotion, and hunger ( $\mathrm{Pa}-$ ulus and Stein 2010). Since such interoceptive information can influence conscious voluntary behavior under some circumstances, it stands to reason that conscious control systems may be utilizing such inputs (Goldstein et al. 2009). As part of this speculation, the aI may be acting as a preferential clearinghouse for interoceptive information, while the dACC preferentially receives more "cognitive", or exteroceptive outputs from other parts of cortex.

If the aI forms part of a potential conduit between somatic states and voluntary control of behavior, it could help explain the fascinating finding that insular lesions increase the likelihood of successfully quitting smoking 100-fold over other brain lesions (Naqvi et al. 2007; Naqvi 
and Bechara 2010). Several patients with insular lesions reported no longer feeling the urge to smoke. If a hypothetical clearinghouse for somatic inputs in the aI were disrupted, it might weaken the influence of autonomic inputs, such as withdrawal effects (Garavan 2010), on consciously controlled behavior.

We view these potential distinctions as preferential, however, in that regions of both the insula and the dACC appear to contribute to an overwhelming percentage of goal-directed tasks.

Acknowledgments We thank Kelly Anne Barnes, Gagan S. Wig, and Bud Craig, as well as an anonymous reviewer for helpful suggestions. We also thank David Van Essen for providing the first version of the map shown in Figure 1. Funding for this work was provided by NIH grants NS61144 (S.E.P.), NS46424 (S.E.P.), NS62489 (A.L.C.), HD57076 (B.L.S.), NS53425 (B.L.S.), NS41255 (B.L.S.) and IGERT NSF548890.

Open Access This article is distributed under the terms of the Creative Commons Attribution Noncommercial License which permits any noncommercial use, distribution, and reproduction in any medium, provided the original author(s) and source are credited.

\section{References}

Albanese M-C, Duerden EG, Rainville P, Duncan GH (2007) Memory traces of pain in human cortex. J Neurosci 27:46124620

Allman JM, Watson KK, Tetreault NA, Hakeem AY (2005) Intuition and autism: a possible role for Von Economo neurons. Trends Cogn Sci 9:367-373

Allman JM, Tetreault NA, Hakeen AY, Manaye KF, Semendeferi K, Erwin JM, Park S, Goubert V, Hof PR (2010) The von Economo neurons in frontoinsular and anterior cingulate cortex in great apes and humans. Brain Struct Funct 214(5-6). doi:10.1007/ s00429-010-0254-0 (this issue)

Berns GS, Chappelow J, Cekic M, Zink CF, Pagnoni G, MartinSkurski ME (2006) Neurobiological substrates of dread. Science 312:754-758

Brass M, Haggard P (2010) The hidden side of intentional action: the role of the anterior insular cortex. Brain Struct Funct 214(5-6). doi:10.1007/s00429-010-0269-6 (this issue)

Butti C, Hof PR (2010) The insular cortex: a comparative perspective. Brain Struct Funct 214(5-6). doi:10.1007/s00429-010-0264-y (this issue)

Canny J (1986) A computational approach to edge detection. IEEE Trans Pattern Anal Mach Intell PAMI 8:679-698

Chua HF, Gonzalez R, Taylor SF, Welsh RC, Liberzon I (2009) Decision-related loss: regret and disappointment. Neuroimage 47:2031-2040

Cohen AL, Fair DA, Dosenbach NU, Miezin FM, Dierker D, Van Essen DC, Schlaggar BL, Petersen SE (2008) Defining functional areas in individual human brains using resting functional connectivity MRI. Neuroimage 41:45-57

Craig ADB (2009) How do you feel-now? The anterior insula and human awareness. Nat Rev Neurosci 10:59-70

Craig AD (2010) The sentient self. Brain Struct Funct 214(5-6). doi: 10.1007/s00429-010-0248-y (this issue)
Dosenbach NUF, Visscher KM, Palmer ED, Miezin FM, Wenger KK, Kang HC, Burgund ED, Grimes AL, Schlaggar BL, Petersen SE (2006) A core system for the implementation of task sets. Neuron 50:799-812

Dosenbach NUF, Fair DA, Miezin FM, Cohen AL, Wenger KK, Dosenbach RAT, Fox MD, Snyder AZ, Vincent JL, Raichle ME, Schlaggar BL, Petersen SE (2007) Distinct brain networks for adaptive and stable task control in humans. Proc Natl Acad Sci USA 104:11073-11078

Dosenbach NUF, Fair DA, Cohen AL, Schlaggar BL, Petersen SE (2008) A dual-networks architecture of top-down control. Trends Cogn Sci 12:99-105

Duncan J, Owen AM (2000) Common regions of the human frontal lobe recruited by diverse cognitive demands. Trends Neurosci 23:475-483

Dunckley P, Wise RG, Aziz Q, Painter D, Brooks J, Tracey I, Chang $\mathrm{L}$ (2005) Cortical processing of visceral and somatic stimulation: differentiating pain intensity from unpleasantness. Neuroscience 133:533-542

Eisenberger NI, Lieberman MD, Williams KD (2003) Does rejection hurt? An FMRI study of social exclusion. Science 302:290-292

Fair DA, Schlaggar BL, Cohen AL, Miezin FM, Dosenbach NU, Wenger KK, Fox MD, Snyder AZ, Raichle ME, Petersen SE (2007) A method for using blocked and event-related fMRI data to study "resting state" functional connectivity. Neuroimage 35:396-405

Fox MD, Snyder AZ, Barch DM, Gusnard DA, Raichle ME (2005a) Transient BOLD responses at block transitions. Neuroimage 28:956-966

Fox MD, Snyder AZ, Vincent JL, Corbetta M, Van Essen DC, Raichle ME (2005b) The human brain is intrinsically organized into dynamic, anticorrelated functional networks. Proc Natl Acad Sci USA 102:9673-9678

Friston K, Jezzard P, Turner R (1994) Analysis of functional MRI time-series. Hum Brain Mapp 1:153-171

Garavan H (2010) The insula and drug cravings. Brain Struct Funct 214(5-6). doi:10.1007/s00429-010-0259-8 (this issue)

Goldstein RZ, Craig ADB, Bechara A, Garavan H, Childress AR, Paulus MP, Volkow ND (2009) The neurocircuitry of impaired insight in drug addiction. Trends Cogn Sci 13:372-380

Gray MA, Rylander K, Harrison NA, Wallin BG, Critchley HD (2009) Following one's heart: cardiac rhythms gate central initiation of sympathetic reflexes. J Neurosci 29:1817-1825

Heimer L, Van Hoesen GW (2006) The limbic lobe and its output channels: implications for emotional functions and adaptive behavior. Neurosci Biobehav Rev 30:126-147

Hester R, Fassbender C, Garavan H (2004) Individual differences in error processing: a review and reanalysis of three event-related fMRI studies using the GO/NOGO task. Cereb Cortex 14:986994

Houde O, Rossi S, Lubin A, Joliot M (2010) Mapping numerical processing, reading, and executive functions in the developing brain: an fMRI meta-analysis of 52 studies including 842 children. Dev Sci. doi:10.1111/j.1467-7687.2009.00942.x

Kavia RB, Dasgupta R, Fowler CJ (2005) Functional imaging and the central control of the bladder. J Comp Neurol 493:27-32

Klein TA, Endrass T, Kathmann N, Neumann J, von Cramon DY, Ullsperger M (2007) Neural correlates of error awareness. Neuroimage 34:1774-1781

Kurth F, Zilles K, Fox PT, Laird AR, Eickhoff SB (2010) A link between the systems: functional differentiation and integration within the human insula revealed by meta-analysis. Brain Struct Funct 214(5-6). doi:10.1007/s00429-010-0255-z (this issue)

Lamm C, Meltzoff AN, Decety J (2010) How do we empathize with someone who is not like us? A functional magnetic resonance imaging study. J Cogn Neurosci 22:362-376 
Lancaster JL, Glass TG, Lankipalli BR, Downs H, Mayberg H, Fox PT (1995) A modality-independent approach to spatial normalization of tomographic images of the human brain. Hum Brain Mapp 3:209-223

Linke J, Kirsch P, King AV, Gass A, Hennerici MG, Bongers A, Wessa M (2010) Motivational orientation modulates the neural response to reward. Neuroimage 49:2618-2625

Medford N, Critchley HD (2010) Conjoint activity of anterior insular and anterior cingulate cortex: awareness and response. Brain Struct Funct 214(5-6). doi:10.1007/s00429-010-0265-x (this issue)

Mesulam MM, Mufson EJ (1982a) Insula of the old world monkey. I. Architectonics in the insulo-orbito-temporal component of the paralimbic brain. J Comp Neurol 212:1-22

Mesulam MM, Mufson EJ (1982b) Insula of the old world monkey. III: Efferent cortical output and comments on function. J Comp Neurol 212:38-52

Michelon P, Snyder AZ, Buckner RL, McAvoy M, Zacks JM (2003) Neural correlates of incongruous visual information. An eventrelated fMRI study. Neuroimage 19:1612-1626

Miezin FM, Maccotta L, Ollinger JM, Petersen SE, Buckner RL (2000) Characterizing the hemodynamic response: effects of presentation rate, sampling procedure, and the possibility of ordering brain activity based on relative timing. Neuroimage 11:735-759

Mufson EJ, Mesulam MM (1982) Insula of the old world monkey. II: Afferent cortical input and comments on the claustrum. J Comp Neurol 212:23-37

Mugler JP III, Brookeman JR (1990) Three-dimensional magnetization-prepared rapid gradient-echo imaging (3D MP RAGE). Magn Reson Med 15:152-157

Mutschler I, Wieckhorst B, Kowalevski S, Derix J, Wentlandt J, Schulze-Bonhage A, Ball T (2009) Functional organization of the human anterior insular cortex. Neurosci Lett 457:66-70

Nanetti L, Cerliani L, Gazzola V, Renken R, Keysers C (2009) Group analyses of connectivity-based cortical parcellation using repeated k-means clustering. Neuroimage 47:1666-1677

Naqvi NH, Rudrauf D, Damasio H, Bechara A (2007) Damage to the insula disrupts addiction to cigarette smoking. Science 315:531534

Naqvi NH, Bechara A (2010) The insula and drug addiction: an interoceptive view of pleasure, urges and decision-making. Brain Struct Funct 214(5-6). doi:10.1007/s00429-010-0268-7 (this issue)

Nimchinsky EA, Gilissen E, Allman JM, Perl DP, Erwin JM, Hof PR (1999) A neuronal morphologic type unique to humans and great apes. Proc Natl Acad Sci USA 96:5268-5273

Ojemann JG, Akbudak E, Snyder AZ, McKinstry RC, Raichle ME, Conturo TE (1997) Anatomic localization and quantitative analysis of gradient refocused echo-planar fMRI susceptibility artifacts. Neuroimage 6:156-167

Ollinger JM, Shulman GL, Corbetta M (2001) Separating processes within a trial in event-related functional MRI I. The method. Neuroimage 13:210-217
Paulus MP, Stein MB (2010) Interoception in anxiety and depression. Brain Struct Funct 214(5-6). doi:10.1007/s00429-010-0258-9 (this issue)

Ploran EJ, Nelson SM, Velanova K, Petersen SE, Wheeler ME (2007) Evidence accumulation and the moment of recognition: dissociating perceptual recognition processes using fMRI. J Neurosci 27:11912-11924

Rossion B, Pourtois G (2004) Revisiting Snodgrass and Vanderwart's object pictorial set: the role of surface detail in basic-level object recognition. Perception 33:217-236

Shaw P, Lalonde F, Lepage C, Rabin C, Eckstrand K, Sharp W, Greenstein D, Evans A, Giedd JN, Rapoport J (2009) Development of cortical asymmetry in typically developing children and its disruption in attention-deficit/hyperactivity disorder. Arch Gen Psychiatry 66:888-896

Snyder AZ (1996) Difference image vs. ratio image error function forms in PET-PET realignment. In: Myer R, Cunningham VJ, Bailey DL, Jones T (eds) Quantification of brain function using PET. Academic Press, San Diego, pp 131-137

Sterzer P, Kleinschmidt A (2010) Anterior insula activations in perceptual paradigms: often observed but barely understood. Brain Struct Funct 214(5-6). doi:10.1007/s00429-010-0252-2 (this issue)

Talairach J, Tournoux P (1988) Co-planar stereotaxic atlas of the human brain. Thieme Medical Publishers, Inc., New York

Thielscher A, Pessoa L (2007) Neural correlates of perceptual choice and decision making during fear-disgust discrimination. J Neurosci 27:2908-2917

Ullsperger M, Harsay HA, Wessel JR, Ridderinkhof KR (2010) Conscious perception of errors and its relation to the anterior insula. Brain Struct Funct 214(5-6). doi:10.1007/s00429010-0261-1 (this issue)

Van Essen DC (2005) A population-average, landmark- and surfacebased (PALS) atlas of human cerebral cortex. Neuroimage 28:635-662

Van Essen DC (2009) Lost in localization-but found with foci?!. Neuroimage 48:14-17

Van Essen DC, Dierker DL (2007) Surface-based and probabilistic atlases of primate cerebral cortex. Neuron 56:209-225

Van Essen DC, Dickson J, Harwell J, Hanlon D, Anderson CH, Drury HA (2001) An integrated software suite for surface-based analyses of cerebral cortex. J Am Med Inform Assoc 41:13591378. See also http://brainmap.wustl.edu/caret

Visscher KM, Miezin FM, Kelly JE, Buckner RL, Donaldson DI, McAvoy MP, Bhalodia VM, Petersen SE (2003) Mixed blocked/ event-related designs separate transient and sustained activity in fMRI. Neuroimage 19:1694-1708

Wheeler ME, Petersen SE, Nelson SM, Ploran EJ, Velanova K (2008) Dissociating early and late error signals in perceptual recognition. J Cogn Neurosci 20:2211-2225 